\title{
The effect of morning vs evening exercise on the temporal distribution of energy and macronutrient intakes, appetite and food preferences: implications for weight management
}

\author{
P.G. Brooker ${ }^{1}$, N.A. King ${ }^{2}$, S.R. Gomersall ${ }^{1,3}$ and M.D. Leveritt ${ }^{1}$ \\ ${ }^{1}$ School of Human Movement and Nutrition Sciences, The University of Queensland, St. Lucia, Queensland, Australia, \\ ${ }^{2}$ School of Exercise \& Nutrition Sciences, Queensland University of Technology, Kelvin Grove, Queensland, Australia \\ and ${ }^{3}$ School of Health \& Rehabilitation Sciences, The University of Queensland, St. Lucia, Queensland, Australia.
}

Temporal variation in the distribution of energy and macronutrient intakes across the day has been associated with overall energy intake $(\mathrm{EI})^{(1)}$. Some evidence suggests that eating more calories later in the day is associated with poorer diet quality and higher body mass index ${ }^{(2)}$. Exercise can alter EI via changes in appetite and food preferences, which may affect the efficacy of exercise in supporting weight loss ${ }^{(3)}$. Before- or after-work are practical times for individuals to incorporate exercise, but it is unknown how eating patterns change when exercise is performed at different times of the day. Therefore, the purpose of this study was to investigate how overweight individuals distribute their EI while participating in an exercise intervention that involves either morning (AM) or evening (PM) sessions.

Forty-three inactive adults (age 41y; females $=69 \% ; \mathrm{BMI}=31 \mathrm{~kg} / \mathrm{m}^{2}$ ) were randomised into one of two 12-week self-paced aerobic exercise programs: AM $(n=21)$, or PM $(n=22)$. EI was measured at baseline, mid-, and post-intervention using a 5-step multiple-pass $24 \mathrm{~h}$ food recall. Absolute daily energy and macronutrient intakes, and temporal distribution of energy and macronutrients, assessed over five periods during the day ${ }^{(1)}$, were calculated. Subjective appetite sensations and food preferences were measured in a fasted state at baseline and post-intervention using 100mm visual analogue scales, and the Leeds Food Preference Questionnaire (LFPQ), respectively.

Both AM and PM groups significantly reduced body mass from baseline to post-intervention $(\mathrm{AM}-2.7, \mathrm{p}=0.01 ; \mathrm{PM}-3.2 \mathrm{~kg}, \mathrm{p}=$ 0.004). EI was significantly reduced from baseline to mid-intervention (AM 7,740 vs 5,523; PM 9,530 vs 6,531 kJ, respectively; p= 0.04), but there were no between-group differences. At the mid-point of the intervention, AM had a higher EI in the lunch period (1000-1359; AM 2,115, PM 1,823 kJ), whereas PM had a higher EI in the dinner period (1800-2159; AM 1,994, PM 2,317 kJ), Fig.1. However, these differences were not significant. There were no between-group differences in the temporal distribution of macronutrient intakes across the five time periods, Fig. 1.
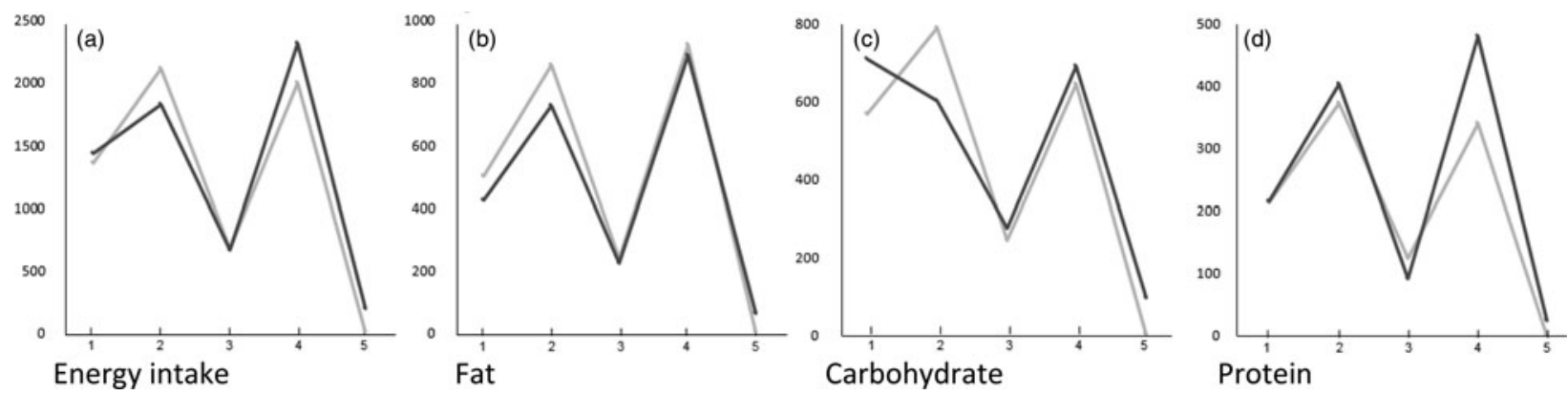

Fig. 1. Temporal distribution of energy (a) and macronutrient (b-d) intakes (y-axis; kJ) across five time periods during the day (x-axis; $1=0600-0959$ $2=1000-1359,3=1400-1759,4=1800-2159$, and $5=2200-0159 \mathrm{~h}$ ) in response to AM (light) and PM (dark) exercise. Data are expressed as group mean.

Both AM and PM groups showed an increased preference for low-fat foods (savoury, $\mathrm{p}=0.012$; sweet, $\mathrm{p}<0.001$ ) and preference for high-fat food decreased (savoury, $\mathrm{p}=0.008$; sweet, $\mathrm{p}<0.001$ ) from baseline to post-intervention, but there were no between group differences.

This study supports previous work that exercise can moderate appetite and influence food choices. Lunch and dinner periods appear to offer compensatory occasions in response to morning and evening exercise, respectively. Since eating later in the day has been associated with increased EI and obesity, exercise timing may play a role in weight management.

1. de Castro JM. (2004) J. Nutr 134 (1), 104-11

2. Leech RM, Timperio A, Livingstone KM et al. (2017) Am J Clin Nutr 106(4), 1121-30

3. Finlayson G, Bryant E, Blundell JE et al. (2009) Physiol Behav 97(1), 62-7 